\title{
GAIA Level 2b Postnatally Diagnosed Congenital Microcephaly
}

National Cancer Institute

\section{Source}

National Cancer Institute. GAIA Level 2b Postnatally Diagnosed Congenital Microcephaly. NCI Thesaurus. Code C128759.

GAIA Level 2b Postnatally Diagnosed Congenital Microcephaly is defined by three criteria: first, a live birth, stillbirth, or spontaneous or therapeutic abortion of at least 24 weeks gestational age (GA), with GA assessed based on uncertain LMP with 2 nd trimester ultrasound (US) scan; second, a head circumference (HC) measurement either 2 standard deviations (SD) below the mean or less than the third percentile as assessed by GA and gender, using appropriate, standardized reference charts for the population (e.g. WHO growth reference charts if GA greater than or equal to 37 weeks and Interg rowth21st reference charts for GA 24 to 36 weeks); third, one of the following requirements is met: a) the measurement is taken within the first 24 hours after birth or at the end of the pregnancy taking into consideration the molding of the head; OR b) the measurement is taken greater than 36 hours and up to 6 weeks after birth or the end of the pregnancy with no apparent post-natal insult resulting in microcephaly. 